\title{
Growth of Cacti-like ZnO nanostructure from aqueous medium for gas sensor application
}

\author{
Rajendra C. Pawar, Jin-Woong Lee, Vikas B. Patil, Caroline S. Lee* \\ ${ }^{1}$ Multi-Functional Materials and Devices Laboratory, Department of Materials Engineering, \\ Hanyang University, ERICA Campus, Ansan, South Korea - 426791. \\ ${ }^{2}$ Materials Research Laboratory, School of physical Sciences, Solapur University, \\ Solapur -413255, (MS), India. \\ Corresponding author's: sunyonglee@hanyang.ac.kr
}

\begin{abstract}
:
Multi-dimensional nanostructure of $\mathrm{ZnO}$ was synthesized by facile solution method at $90{ }^{\circ} \mathrm{C}$. The scanning electron microscopy (SEM) images of these films shows vertically aligned $\mathrm{ZnO}$ nanoneedles and globe cacti-like morphology. X-ray diffraction (XRD) pattern of these nanoneedles shows highly crystalline $\mathrm{ZnO}$ along (002) direction with wurtzite crystal structure. The synthesized $\mathrm{ZnO}$ nanostructures are used for NO2 sensing properties at different operating temperatures and gas concentrations. The cacti-like structure exhibits higher gas response at low operating temperature $\left(85 \%\right.$ at $\left.275{ }^{\circ} \mathrm{C}\right)$ as compared with nanorods $\left(71 \%\right.$ at $\left.325^{\circ} \mathrm{C}\right)$ upon exposure to NO2 gas. The high sensitivity and low operating temperature is attributed to the small size of secondary branches. Additionally, the cacti-like structure is able to respond quickly upon the exposure of NO2 gas. These results show that the cactus-like structure is promising for NO2 gas sensor application at low temperature.
\end{abstract}

Key words: Zinc oxide, gas sensor, aqueous chemical method

\section{Introduction}

Over the past several decades, wide band gap semiconductors have been extensively investigated due to their potential applications in gas sensor, solar cells and other devices [1]. $\mathrm{ZnO}$ is one of the most important materials due to its unique properties like piezoelectricity, optical absorption, chemical stability, catalytic activity, high voltage-current nonlinearity and emission [2,3]. Further, its bio-compatibility and safety make suitable in biomedical applications [4]. It has wide range of applications including room-temperature UV lasers, light-emitting diodes, piezoelectric and chemical sensors, catalysis, actuators, varistors, photodetectors and photonic crystals.

Though variety of $\mathrm{ZnO}$ nanostructures has been synthesized, only few of species have been employed in gas sensor studies. The morphologies of $\mathrm{ZnO}$ nanoparticles, nanorods, nanobeads, aligned nanorods, cabbage leaf have been investigated for their gas sensor properties. The gas response and operating temperature of the sensor is mainly depends on materials active surface area, porosity, stoichiometry, defects concentration, crystal orientation, catalytic activity, bond ionicity and work function [5,6]. Three dimensional (3D) hierarchical branching nanowire structures offer a better opportunity while in gas sensor because of large specific surface area [7]. The addition of branching to array of 1D improves oxygen adsorption, which may increase the interaction between oxygen and material [8]. Therefore, 3D nanostructures are more applicable for gas sensor application.

In the present work, authors have synthesized a novel cacti-like structure of $\mathrm{ZnO}$ using aqueous chemical method at $90{ }^{\circ} \mathrm{C}$. The $\mathrm{NO} 2$ gas sensing characteristics has been discussed. Further the relation between $\mathrm{NO} 2$ gas sensing and nanostructures is studied in detail.

\section{Experimental}

The novel cacti-like structures were prepared using two step processes. Initially seed solution was prepared in an absolute ethanol with 0.05 $M$ zinc acetate $\left(\mathrm{Zn}\left(\mathrm{CH}_{3} \mathrm{COO}\right)_{2} \cdot 2 \mathrm{H} 2 \mathrm{O}\right)$ and 0.05 $M$ diethanolamine $\left(\mathrm{HN}\left(\mathrm{CH}_{2} \mathrm{CH}_{2} \mathrm{OH}\right)_{2}\right.$, DEA). The cleaned glass substrate was dip coated for $10 \mathrm{~s}$ in a seed solution and then kept at room temperature over night for drying. The dried films were annealed at $400{ }^{\circ} \mathrm{C}$ for $5 \mathrm{~min}$ in air to 
yield a layer of $\mathrm{ZnO}$ seed on the substrate. The seeded substrates were placed vertically in 200 $\mathrm{ml}$ solution (growth solution) containing an equimolar $(0.05 \mathrm{M})$ zinc acetate and hexamethylenetetramine (HMTA) and refluxed at $95 \pm 3{ }^{\circ} \mathrm{C}$ for $5 \mathrm{~h}$. The refluxed film containing NRs array was re-seeded and re-refluxed at 90 ${ }^{\circ} \mathrm{C}$ for $5 \mathrm{~h}$ in growth solution, to form $\mathrm{ZnO}$ cactilike nanostructures.

\section{Results and discussion}

The XRD patterns of nanorods and cacti-like nanostructured films on the glass substrate (Fig 1) indicate the formation of highly crystalline $\mathrm{ZnO}$ phase with a hexagonal (wurtzite) crystal structure (JCPDS card no 76-0704). Nanorods grown film exhibits highly oriented peak along (002). Whereas $\mathrm{ZnO}$ cacti exhibits two minor peaks along (101) and (011) plane in addition to a major peak along (002). The inset shows the initial orientation of $\mathrm{ZnO}$ seed along (002) plane.

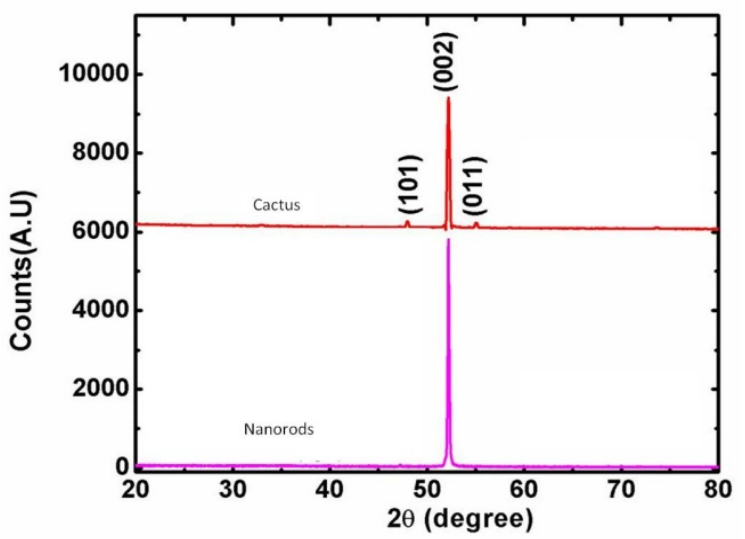

Fig.1. XRD pattern of Nanorods and Cacti-like structures.

The SEM micrograph demonstrates the $\mathrm{ZnO}$ nanorods and their uniform distribution over the substrate (Fig. 2(a)). Their average rods diameter is about $70 \mathrm{~nm}$. Fig. 2(b) shows SEM micrograph of cacti structure. Highly dense nanorods are eminated from single microrod. This secondary nanorods are grown from single nucleation centres attached in second time seeding. The average diameter of $80 \mathrm{~nm}$ and length of $1.5 \mu \mathrm{m}$ is observed for secondary nanorods.

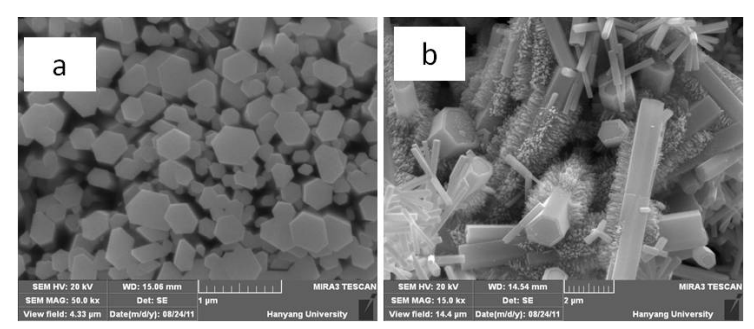

Fig.2. FESEM images of Nanorods and Cacti-like structures
The gas response of nanorods and cacti-like structures was measured for NO2 over 200$450{ }^{\circ} \mathrm{C}$. Response for both films is found to be similar, i.e. the gas response increases with the operating temperature, attains maximum value and then decreases with a further rise in operating temperature. It is seen that, at the same $\mathrm{NO} 2$ concentration, the $\mathrm{ZnO}$ sensors shows different responses for different morphologies. The NO2 gas response is higher at an optimum operating temperature ( $\left.T_{O P}\right)$ of the film and it is lower on either side of $T_{O P}$. However, the magnitude of gases, and $T_{O P}$ vary for the both samples due to their different morphologies. Table 1 shows $\mathrm{T}_{\mathrm{OP}}$ and their related value of the gas response. Cactus structure exhibits a higher gas response at relatively lower value of $T_{O P}$. High response and low $T_{O P}$ of cactus structure are related to the size and shape of $\mathrm{ZnO}$ nanostructures [9]. The higher gas response in attributed to (i) higher surface to volume ratio of secondary nanorods provides more surface area to interact the NO2 gas molecules, (ii) Improvement in number of adsorbed oxygen molecules and (iii) diameter of secondary nanorods is the order of Debye length, hence increased change in resistance $(\Delta \mathrm{R})$ due to nanorrowing of channel width.

\begin{tabular}{|c|c|c|}
\hline Sample Code & ToP in ${ }^{\circ} \mathbf{C}$ & $\begin{array}{c}\text { Gas Response } \\
\text { in \% }\end{array}$ \\
\hline Nanorods & 325 & 71 \\
\hline Cactus & 275 & 85 \\
\hline
\end{tabular}

Table 1: The NO2 gas response at optimum operating temperatures.

The response time $\left(R_{P}\right)$ is defined as the time taken by sensor to attain $90 \%$ of the maximum decrease in resistance on exposure to the target gas. The recovery time $\left(R_{C}\right)$ is defined as the time taken by the sensor to get back $90 \%$ of original resistance. The dynamic variation of response for cactus-like structure at $100 \mathrm{ppm}$ $\mathrm{NO} 2$ is shown in Fig. 3. In these measurements, gas was introduced into the sealed glass tube and measured the resistivity of sensors in presence of air and NO2. Fast response and recovery time is observed. The sensor response returned to its initial value after the pumping of NO2 gas away which indicates the surface of $\mathrm{ZnO}$ nanostructures remains same after the pumping of $\mathrm{NO} 2$ gas.

For n-type semiconductor, it is known that the chemisorption of oxygen on a zinc oxide surface captures electron from conduction band and hence reduce the conductance. This forms the depletion layer in the surface of $\mathrm{ZnO}$ nanostructures, causing decrement in the carrier concentration [10]. Therefore the 
resistance of $\mathrm{ZnO}$ in ambient air is higher than that in vacuum. When $\mathrm{NO} 2$ is introduced at optimal operating temperature,

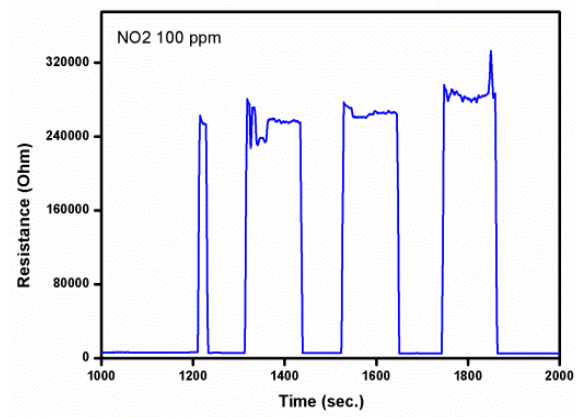

Fig.3. Gas response of NO2 for Cacti structure at $100 \mathrm{ppm}$

the NO2 molecules will react with the adsorbed $\mathrm{O}^{-}$or $\mathrm{O}^{2-}$, releasing the trapped electrons back to the conduction band, and therefore the conductivity of the $\mathrm{ZnO}$ sensors increases.

\section{Conclusions}

In summary, we have successfully synthesized a novel cacti-like $\mathrm{ZnO}$ structure on glass substrates with the aqueous chemical method at relatively low temperature $\left(90^{\circ} \mathrm{C}\right)$. It is observed that the sensors based on cactus structure showed higher response $(85 \%)$ for $\mathrm{NO} 2$ gas at $275^{\circ} \mathrm{C}$. The higher gas response is explained in terms of the Debye length and the entire surface contact between the structure. Our results demonstrate that the cacti-like structure has excellent potential applications in low temperature NO2 gas sensors.

\section{Acknowledgement}

This research is supported by basic science research program through National Research Foundation of Korea (NRF) funded by the Ministry of Education, Science and Technology (2011-0027380) and Mid-career Researcher Program through NRF grant funded by the MEST (no. 2011-0000201).

\section{References}

[1] A. Tricoli, M. Righettoni, A. Teleki, Angew. Chem. Int. Ed. 49, 7632-7659 (2010); doi:10.1002/anie.20-0903801

[2] Z.L. Wang, Mater. Sci. Eng. R 64, 33-71 (2009); doi:10.1016/j.mser.2009.02.001

[3] U. Ozgu, Y.I. Alivov, C. Liu, A. Teke, M.A. Reshchikov, S. Dogan, V. Avrutin, S.J. Cho, H. Morkocd, J. Appl. Phys. 98, 041301-041402 (2005); doi:10.1063/1.1992666

[4] A.E. Shafei, A.A. Okeil, Carbohydrate Poly. 83, 920-925 (2011); doi: 10.1016/j.carbpol.2010. 08.083

[5] Z. Jing, J. Zhan, Adv. Mater. 20, 4547-4551 (2008); doi:10.1002/adma.200800243

[6] J. Janata, Chemical Sensors, Anal. Chem. 62, 33R-44R (1990); doi: 10.1021/ac00211a017

[7] J. Bae, J. Hong, W.H. Han, Y.J. Choi, R.L. Snyder, Chem. Phys. Lett. 475, 260-263 (2009); doi: 10.1016/j.cplett.2009.05.045

[8] A.I. Inamdar, S.H. Mujawar, V. Ganesan, P.S. Patil, Nanotechnology 19, 325706-325712 (2008); doi: 10.1088/0957-4484/19/32/325706

[9] B. Baruwati, D.K. Kumar, S.V. Manorama, Sens. Actuators B: Chem. 97, 49-58 (2004); doi:10.1016/j.-snb.2003.06.003

[10] A. Rothschild, Y, Komem, J. Appl. Phys. 95, 6374-6380 (2004); doi:10.1063/1.1728314 